\title{
ORGANIZACIÓN DE DOCENCIA E INVESTIGACIÓN EN UNIVERSIDADES: UNA EXPLORACIÓN AL CASO CHILENO
}

\section{Daniel Theurillat y Benjamín Gareca ${ }^{1}$}

\begin{abstract}
RESUMEN
La investigación busca indagar en la forma en que las universidades chilenas articulan su actividad investigativa y docente, ya que se ha demostrado que no siempre conviven de forma armónica, lo cual puede provocar dificultades y tensiones dentro de la organización institucional. Para ello, se implementó un estudio cualitativo exploratorio con entrevistas semiestructuradas en profundidad a funcionarios relevantes de distintas instituciones universitarias, correspondientes a diversas ramas del conocimiento. Con la información recabada, se formó una caracterización preliminar de las universidades chilenas respecto de su investigación y docencia. Entre los principales resultados se observa que existen diferencias en relación con las siguientes dimensiones relativas a la investigación y docencia: distribución, criterios de promoción, formas de evaluación e incentivos. En este sentido, las universidades más complejas tienden a presentar estándares más rígidos para estas dimensiones. Además, se concluye que el sistema chileno, en general, tiene un sesgo a valorar en mayor medida la investigación, de manera similar a lo que ocurre en el mundo industrializado.
\end{abstract}

Palabras clave: investigación, docencia, organización, educación superior, académicos.

\section{ORGANIZATION OF TEACHING AND RESEARCH AT UNIVERSITIES: AN EXPLORATION OF THE CHILEAN CASE}

\begin{abstract}
The current investigation aims to explore the manner in which Chilean universities articulate their teaching and research activities. This is relevant as it has been shown that these activities do not always coexist harmonically, which can generate difficulties and tensions within the institutional organization. To approach this, a qualitative exploratory study was implemented, consisting of indepth semi-structured interviews of relevant institutional officials from diverse universities, belonging to different disciplines of knowledge. With the gathered information, a preliminary characterization of Chilean universities was constructed in reference to research and teaching. Among the main results, it can be observed that there are differences in regards to the following dimensions related to research and teaching: distribution of activities; promotion criteria; evaluation formats and incentives. In this sense, the more complex universities tend to present more rigid standards for the aforementioned dimensions. In addition, it has been concluded that the Chilean higher education system, in general, has a tendency to hold research in higher esteem, as occurs in industrialized countries.
\end{abstract}

Keywords: research, teaching, organization, higher education, academics.

1 Pontificia Universidad Católica de Chile, Santiago, Chile. Contacto: dtheuri@@uc.cl y bogareca@uc.cl 


\section{Introducción}

El Centro de Estudios de Políticas y Prácticas en Educación (CEPPE) solicitó la realización del presente estudio con la finalidad de indagar en la tensión que podría surgir en torno a la organización de las prácticas de investigación y docencia universitarias en Chile. Esta necesidad se origina porque en el contexto global se ha hecho evidente que las exigencias sociales hacia las universidades han aumentado considerablemente. Por un lado, se espera que realicen docencia de calidad y a una población de estudiantes cada vez mayor y, al mismo tiempo, que se sitúen como instituciones encargadas de realizar investigación de vanguardia para potenciar el desarrollo económico, político y social del país.

Chile no ha sido excepción en estos fenómenos, pues especialmente desde los años ochenta el ingreso a la educación superior se ha incrementado notablemente y, al mismo tiempo, la nueva estructura de financiamiento ha obligado a las universidades a posicionar la investigación en el centro de su actividad. Dado este contexto, sería esperable observar tensiones entre estas exigencias, no obstante, no se ha abordado de manera concreta la problemática sobre la base de evidencia empírica.

Por tanto, el objetivo general de esta investigación fue indagar en el modo en que las instituciones de educación superior chilenas organizan docencia e investigación, en búsqueda de posibles nodos críticos en su relación. Este esfuerzo es relevante en la medida en que permite explorar la estructura organizacional de las instituciones de educación superior, diagnóstico que podría otorgar lineamientos para una mejor conjugación de ambas actividades.

\section{Discusión bibliográfica}

\subsection{Procesos sociales precedentes}

Como se señalaba más arriba, la tensión entre docencia e investigación en instituciones de educación superior surge de dos procesos distintos: la masificación del acceso a ella y el aumento de las expectativas sociales respecto de su investigación (Clark, 1998). Ambos procesos 
se manifiestan en los modos según los cuales los establecimientos de educación superior obtienen recursos y, mediante ello, organizan sus actividades, estructuras, jerarquías, recompensas y misiones, entre otros factores.

\subsubsection{Masificación}

El primer proceso, la masificación, se relaciona con un aumento exponencial y significativo en la cantidad y pluralidad de los matriculados y de las instituciones (Altbach, Reisberg \& Rumbley, 2009). Esto surge de la necesidad de satisfacer la creciente demanda de educación superior, que a su vez se debe a la expansión del sector terciario, y su requerimiento del manejo de tecnología y habilidades cada vez más específicas. En este contexto, la educación se entiende como perentoria para la consecución de la movilidad y el éxito socioeconómico. Todo lo anterior lleva a nuevos patrones de financiamiento, una mayor diversificación de la oferta y a una disminución de los estándares académicos.

En términos concretos, casi todos los países han incrementado drásticamente sus tasas de participación en la educación superior, sobre todo aquellos en vías de desarrollo (Altbach, Reisberg \& Rumbley, 2009). Globalmente, dentro de la cohorte de edad a la que corresponde atender en educación superior ${ }^{2}$, ha habido un incremento de participación desde un 19\% en 2000 a un 26\% en 2007. En Latinoamérica, el enrolamiento sigue siendo menos de la mitad del existente en países de altos ingresos; no obstante, sí se ha masificado, pues su matrícula ha aumentado un 40,1\% entre 2004 y 2008 (Cinda, 2011).

\subsubsection{Expectativas de investigación}

Por otro lado, en el contexto de una economía global basada en el conocimiento, la presión sobre la producción de investigación ejercida por parte de gobiernos y sociedades ha ido en aumento, cuestión que se contrapone con el rol vocacional o de formación tradicional de estas

2 Tasa bruta de escolaridad: medida relativa (no absoluta) que da cuenta de la masificación respecto de la población nacional. 
instituciones (Leisyte, Enders \& de Boer, 2007; Altbach, Reisberg \& Rumbley, 2009).

En búsqueda de mejorar la eficiencia, productividad y transparencia en la administración de recursos públicos, variados sistemas nacionales de educación superior e investigación han sufrido reformas de diversa magnitud para alentar la investigación (Auranen $\&$ Nieminen, 2010; De Boer, Enders, \& Schimank, 2007; Enders, Kehm, \& Schimank, 2015; Geiger, 2004; Leisyte, Enders \& De Boer, 2007). La masificación, que ha llevado a la escasez de fondos públicos y al cuestionamiento respecto de la sustentabilidad o rentabilidad de las instituciones, ha suscitado parte de estas reformas, las que han obligado a las universidades a competir y a articular su administración en torno a dichos fondos: estudiantes, subvenciones de investigación y cuerpo académico de excelencia se han transformado en objetos de disputa.

\subsection{Organización de investigación y docencia en el contexto global}

\subsubsection{Organización nacional de sistemas universitarios}

Transversalmente, los sistemas se han reformado en mayor o menor medida según el paradigma de la Nueva Administración Pública (NPM, por sus siglas en inglés), modelo que supone que los sistemas que utilizan incentivos a los resultados y mecanismos de competencia son más eficientes y productivos que aquellos que no lo hacen, distribuyendo mayor cantidad de recursos entre quienes tienen mejor desempeño (Auranen \& Nieminen, 2010; De Boer, Enders \& Schimank, 2007; Enders, Kehm \& Schimank, 2015; Geiger, 2004; Marginson, 2006).

Geiger (2004), quien observa el caso de EE.UU., indica que la evolución del sistema a un cuasimercado habría permitido una estrecha vinculación entre los costos y las funciones de la universidad, limitando la discrecionalidad de los administradores. En general, considera que la investigación se habría adaptado y expandido correctamente gracias a los nuevos incentivos de mercado, y que este a su vez habría distribuido los recursos eficientemente tanto a 
alumnos como investigadores de acuerdo con sus capacidades ${ }^{3}$. La expansión de la investigación solo habría sido posible, además, por su separación de la docencia.

Por su parte, De Boer, Enders \& Schimank, (2007) y Enders, Kehm \& Schimank (2015) investigan los casos de Inglaterra, Austria, Holanda y Alemania. Sucintamente, concluyen que las reformas llevadas a cabo alentarían una mayor productividad, pero también producirían un foco más cortoplacista. Al mismo tiempo, estas ayudarían a separar los ámbitos investigativos y docentes, lo que llevaría a una diferenciada distribución de recursos. Esto último es relevante en la medida en que la investigación es más fácilmente conmensurable que la docencia, lo que en general devalúa a la segunda en términos de prestigio y estatus en los países que refuerzan esta distinción (Deem y Lucas, 2007; Horta, Dautel \& Veloso, 2012; Jenkins, 2004; Marginson, 2006).

Por otro lado, Auranen y Nieminen (2010) analizan a ocho naciones industrializadas, y observan que, independientemente de las reformas pro-NPM que se hayan efectuado en los últimos 25 años (1981-2006), la eficiencia (gasto en I\&D/publicaciones) se ha mantenido estable en la mayoría de los países. Aún más, países "poco competitivos" (a saber, con un sistema de financiamiento basal de las universidades) como Suecia y Alemania serían los únicos que han mostrado mejorías. La ventaja mantenida de estos y otros países se podría deber a diferencias de idioma y culturales, antes que a su estructura de financiamiento.

En esta línea, se podría agregar la noción de bienes posicionales (Hirsch, 1976) adoptada por Marginson (2006) como un elemento central en la estructuración de las ventajas de ciertos países sobre otros (y, al interior de los países, de ciertas universidades por sobre otras). En este sentido, una cantidad definida de universidades de elite en ciertos países atraería a alumnos aventajados socioeconómicamente, los cuales pagarían altas matrículas para poder ingresar a dichas

3 El autor reflexiona acerca de las inequidades que reproduce el sistema, tanto a nivel de habilidad de los estudiantes como respecto de los recursos de investigación, pero en suma se inclina a la "virtud" de adaptar los mayores recursos a quienes tienen mayores competencias. 
instituciones y, al mismo tiempo, enaltecerían el nivel de sus cursos por su elevado capital cultural y social. El alto precio de las matrículas permitiría el pago de profesores altamente capacitados, los cuales realizarían investigación de vanguardia, atrayendo más alumnos y profesores. Fuera del círculo de universidades prestigiosas, las otras instituciones solo competirían por llenar sus cupos, única fuente de ingresos permanente. No tendrían capacidad para realizar investigación de modo relevante, por lo que quedarían en una posición intermedia como especialistas, o bien, en el peor de los casos, como instituciones poco selectivas, relegadas "meramente" a la docencia. Así, la investigación sería un mecanismo para reproducir un privilegio y, consecuentemente, su relación con la docencia sería espuria.

\subsubsection{Organización institucional de académicos}

Además de transformar las prioridades y naturaleza de los sistemas universitarios, los procesos de masificación de la matrícula y presión sobre el desarrollo de la investigación habrían traído consigo una reformulación de la actividad académica desarrollada dentro de las instituciones. Los nuevos modelos de financiamiento nacional basados en escasez, competencia y excelencia investigativa incitarían a las universidades a integrar la investigación como un nodo fundamental de su definición institucional.

Sin embargo, con ello se habría expuesto a los académicos a las fuerzas del mercado, obligándolos a situar sus esfuerzos en torno a actividades competitivas que permitieran atraer a sus instituciones los fondos que ahora requieren, y donde la principal recompensa sería el prestigio profesional derivado fundamentalmente de los resultados en investigación (Bernasconi, 2008; Berríos, 2008a; Serow, 2000).

Este giro se hace especialmente evidente en el establecimiento de sistemas institucionales de recompensas, pues ellos constituirían el marco regulador de exigencias, expectativas e incentivos que guía el comportamiento del académico, especialmente durante sus primeros años de trabajo (Berríos, 2008a; Bernasconi, 2008; Diamond, 1993; Link, Swann \& Bozeman, 2008). En concordancia, se ha argumentado que las universidades han instalado un modelo normativo de ideal académico bajo la herramienta principal de la carrera académica, cuyo 
reglamento establece los “...requisitos, criterios y procedimientos para la evaluación de desempeño de los individuos, distribuyéndolos jerárquicamente mediante rangos en el sistema de prestigio [...] [y remuneraciones] de la institución" (Berríos, 2008b, p. 43). Por ejemplo, se ha reportado que, a pesar de que se establecen exigencias mínimas tanto de investigación como de docencia a todos los académicos de jornada completa, los requerimientos para la promoción de los individuos otorgan mayor importancia a productos investigativos clásicos, tales como la publicación en revistas indexadas y la participación en proyectos internacionales: invertir tiempo en docencia, creatividad o servicio termina siendo una actitud riesgosa para aquellos que buscan promoción u obtención de incentivos institucionales (Diamond, 1993). En el mismo sentido, invertir más tiempo en docencia que en investigación se relaciona directamente con la obtención de salarios menores en el mediano y largo plazo (Fairweather, 2005).

En este punto, las universidades de investigación suelen darle un énfasis especialmente importante al producto investigativo, pues cuentan con la expectativa de que sus académicos puedan consolidarse como líderes nacionales e internacionales en sus áreas de conocimiento (Leisyte, Enders \& De Boer, 2009). En tanto, las universidades docentes permiten mayor flexibilidad en la exigencia investigativa, contando como válidas publicaciones en libros, artículos de diario, talleres creativos y publicaciones en medios populares (Colbeck, 1998).

La divergencia disciplinaria también juega un rol importante en el matiz de estas tendencias. Aquellas disciplinas que exigen altos consensos en sus productos (ciencias "duras") generan académicos altamente especializados y dedicados fuertemente a una línea investigativa, mientras que disciplinas de menor exigencia consensual permiten un involucramiento más flexible en la investigación (Colbeck, 1998; Griffiths, 2004; Healey, 2005; Horta, Dautel \& Veloso, 2012).

Ante esto, ciertas corrientes han luchado por reivindicar los demás aspectos de la vida académica. Por un lado se ha llamado a reestructurar los sistemas de recompensa para incluir una nueva 
definición de academia, que se relacione con el conocimiento tanto en su búsqueda (investigación), como en su traspaso (docencia) y aplicación (extensión y servicio) (Colbeck, 1998; Diamond, 1993; Fairweather, 2005; Gotlieb, 1997). Por otro lado, se ha argumentado que debe valorarse la especificidad de la misión determinada por cada institución, cuya tradición la posicionará de forma única ante las actividades de la academia (Fairweather, 2005). Por tanto, los sistemas de recompensas deben poder demostrar que lo que es valorado por las instituciones formalmente será también apreciado en todos los puntos de la promoción y carrera académica (Diamond, 1993; Fairweather, 2005).

A partir de estas preocupaciones, diversas universidades de investigación en el mundo han comenzado a crear premios e incentivos especializados para profesores, establecido centros de enseñanza y aprendizaje, determinado estándares docentes mínimos de promoción y aumentado la carga docente de los académicos (Serow, 2000). Aún así, el salario y la promoción continúan dependiendo en mayor medida de la producción investigativa: a pesar del reposicionamiento de la docencia en la actividad académica a nivel discursivo, la investigación no ha perdido su predominio (Serow, 2000). De hecho, se ha reportado que la docencia solo es atendida cuando es practicada deficientemente. Su evaluación es más bien una prevención de deterioro excesivo que un incentivo a la excelencia (Serow, 2000).

La distribución del tiempo de los académicos de universidades de investigación refleja estas tendencias. En promedio, en periodo de clases, $38 \%$ a $46 \%$ del tiempo de los académicos contratados a jornada completa ${ }^{4}$ se utiliza en docencia, y $28 \%$ a 32\% en investigación (Teichler, Arimoto \& Cummings; 2013). Fuera de este periodo, la mayor parte del tiempo se usa en lo segundo. Finalmente, la mayoría del tiempo de trabajo entre ambos periodos se utiliza en investigación. Sin embargo, esto varía en países emergentes, donde la docencia suele tener más espacio.

4 A nivel mundial, entre el $70 \%$ y el $90 \%$ de los académicos universitarios trabaja jornada completa (Teichler, Arimoto y Cummings, 2013). Sin embargo, esta cifra solo llega al 19\% en Chile (González, Brunner y Salmi, 2013). 


\subsection{Caso chileno}

\subsubsection{Historia y especificidad del contexto educacional chileno} Chile, como gran parte de los países desarrollados o en vías de desarrollo, ha transitado de un sistema elitista, estatal y homogéneo, a uno masificado, de mercado y diverso. Este modelo se inició en parte con la Reforma Universitaria de 1967, que buscaba mayor democratización y equidad de acceso, además de una potenciación del trabajo de investigación, que hasta el momento era prácticamente inexistente (Bernasconi y Rojas, 2003).

Luego del Golpe de Estado, hubo un giro en la política educacional. Hasta 1980, el gasto público en educación disminuyó entre 15\% y 35\%, lo que obligó a las universidades a cobrar aranceles y eliminar la gratuidad. Dada la escasez de recursos y la aparición de instituciones informales para satisfacer la creciente demanda fue necesaria la reforma realizada en 1981 que, con miras a estimular la competencia (bajo la lógica del NPM), permitió la incorporación al sistema de nuevas universidades privadas e instituciones no universitarias de educación superior (institutos profesionales y centros de formación técnica), cuyo crecimiento ha sido asombroso: actualmente este sector corresponde al 75\% (891.000 estudiantes) del total de la matrícula de las instituciones de educación superior del país (1.173.000), y al $100 \%$ del sector no universitario de la educación superior (487.000). Con esta reestructuración la tasa bruta de escolaridad ha aumentado de un 7\% en 1967 a un 55\% en 2012 (Brunner, 1986; OCDE, 2013).

Ahora bien, al igual que en Latinoamérica y en el mundo en general, se ha debido responder a la masificación de la educación superior en un periodo de tiempo breve, contratando cuerpo netamente docente bajo presión, sin los recursos necesarios ni el personal suficientemente calificado. En contrapartida, quienes se han dedicado a la academia normalmente son sujetos más experimentados, aventajados y/o privilegiados (Bernasconi y Rojas, 2003; Cinda, 2011), los cuales además corresponderían a una minoría, pues solo un $6,3 \%$ se ajustarían a la definición de académico (poseer jornada completa y doctorado) propuesta por Bernasconi (2010), según los datos de González, Brunner y Salmi (2013). Adicionalmente, es 
relevante recordar la excepcionalidad del caso chileno respecto de su baja dotación de académicos jornada completa (Teichler, Arimoto \& Cummings, 2013; González, Brunner y Salmi, 2013).

\subsubsection{Sistema de financiamiento}

El hecho de que se premie a profesores investigadores no es fortuito, en la medida en que dos componentes ${ }^{5}$ del sistema chileno de financiamiento anual de la educación superior se relacionan con investigación (Bernasconi y Rojas, 2003). El primero es el Aporte Fiscal Directo (AFD), que brevemente consiste en un subsidio de libre disponibilidad asignado a instituciones en un 95\% según criterios históricos y en un $5 \%$ de acuerdo con indicadores de desempeño (que al año siguiente se vuelven el criterio histórico). De estos indicadores existen dos que nos atañen: el número de proyectos financiados por el Fondo Nacional de Ciencia y Tecnología (Fondecyt) y el número de publicaciones indexadas en revistas ISI, ambos respecto del número de jornadas completas equivalentes. En conjunto, suman el 60\% de los indicadores de desempeño.

El segundo componente atingente a nuestra problemática corresponde a los aportes directos de fondos para la investigación, canalizados a través de la Comisión Nacional de Ciencia y Tecnología, Conicyt, entre los que se encuentran el Fondecyt, el Fondef y otros programas. La fuerza de esto reside en que otorgan independencia económica a los investigadores, liberando fondos de las universidades y además aumentando su prestigio.

\subsubsection{Distribución efectiva de prácticas}

Al atender a la distribución efectiva de la investigación y la docencia en las universidades chilenas, puede señalarse que "[l]a profesión académica propiamente tal ha sido una cuestión principalmente desarrollada en el grupo de universidades de mayor trayectoria y desarrollo de la investigación y el posgrado" (Bernasconi y Rojas, 2003, p. 221). Concretamente, de hecho, solo tres de ellas la

5 El resto de los componentes son el Aporte Fiscal Indirecto (AFI), las Ayudas Estudiantiles, el Fondo de Desarrollo Institucional y otros tipos de financiamiento (Contraloría General de la República). En la práctica son irrelevantes para nuestra investigación. 
realizan a cabalidad. Se puede conjeturar que, si bien existe un efecto acumulativo en el AFD que fue relevante en los inicios del sistema de financiamiento como tal, en la actualidad aquellas universidades que históricamente se han dedicado a la investigación tienen ventajas de personal e infraestructura que se han transformado en insalvables para las universidades exclusivamente docentes, por lo que han abandonado la competencia por dichos recursos. Es necesario tener presente también que las universidades privadas no tradicionales, por ley, no pueden acceder al AFD, con lo que pierden parte importante del aliciente para realizar investigación.

A pesar de lo anterior, se ha argumentado que la carrera académica en universidades chilenas, independientemente del nivel investigativo, se ha incentivado y evaluado bajo criterios investigativos (Berríos, 2008b). Con ello, la idealización del académico investigador se ha establecido como el referente al que toda institución aspira.

En síntesis, dada la demostrada masificación, la preferencia y privilegios hacia la investigación y la importante competencia por la subvención entre algunas de las instituciones, Chile se presenta como un caso ejemplar en donde se podrían estar desplegando múltiples tensiones entre investigación y docencia en la organización de las actividades al interior de la universidad.

\section{Metodología}

Para analizar la relación entre docencia e investigación y sus posibles nodos de conflicto, se delimitaron cinco áreas relevantes de análisis y caracterización.

\subsection{Variables relevantes}

2.1.1. Definiciones de docencia e investigación

El primer factor relevante es el rol de la investigación y la docencia en la misión de las instituciones observadas. En este punto, se busca determinar la importancia declarada de la investigación y la docencia, de modo de poder compararlas con cómo efectivamente son llevadas a la práctica. 


\subsubsection{Carga docente e investigativa}

Dentro de este ámbito, se buscó determinar la cantidad de docentes que se dedica a la docencia y a la investigación, exclusivamente o en conjunto. Por otro lado, se apuntó a determinar la distribución del tiempo de cada académico para ambas actividades. Por último, se indagó acerca de la proporción de académicos contratados en los distintos tipos de jornada.

\subsubsection{Promoción de la carrera académica}

Aquí se sondeó la forma en que las instituciones estructuran la promoción de una carrera académica. Para ello se evaluaron los criterios que se utilizan para la promoción de individuos y cuál es el rol que juegan en ellos la investigación y docencia. Se juzgó qué actividades o desempeños traen mejores expectativas de ascenso o de aumento en su remuneración y, de acuerdo con ello, qué actividades son incitadas bajo estos mecanismos.

\subsubsection{Sistemas de evaluación}

Se buscó determinar los criterios que definen un buen desempeño en la docencia y la investigación. Respecto de la primera, se indagó acerca del rol de la evaluación docente por parte de los alumnos y de la facultad misma, y sobre la existencia de otros factores que la propia universidad considere fundamentales. Acerca de la segunda, se preguntó por aquellos estímulos relacionados con la postulación exitosa a fondos y sobre todo aquellos relacionados con el número y tipo de publicaciones realizadas por los académicos.

\subsubsection{Sistemas de incentivos}

Finalmente, se estableció como meta revelar de qué manera se utilizan estímulos en el fomento de la docencia y de la investigación. Específicamente, se indagó acerca de la existencia de políticas organizacionales orientadas a aumentar la cantidad y calidad de ambas actividades (incentivos de bonificación, por ejemplo). Por último, se inquirió sobre la percepción de los funcionarios entrevistados respecto de los efectos de tal sistema de incentivos en las orientaciones de los académicos hacia estas actividades. 


\subsection{Diseño metodológico}

\subsubsection{Recolección de datos}

Dada la escasa literatura para el caso chileno se diseñó un estudio cualitativo exploratorio, el cual dispuso de dos metodologías de recolección. En primer lugar, se realizó una revisión exhaustiva de documentación pública referente al nivel institucional general de las universidades. Con ello se buscó determinar cuáles son las definiciones formales de investigación y docencia, como también su rol asignado dentro de la organización, esbozados en declaraciones de misión, visión o currículo, entre otros. Además, se buscó recopilar información concreta respecto de la organización de estas actividades, como la dotación docente por tipo de jornada, el número de alumnos por programa, la estructura organizacional interna, la cantidad y el tipo de investigación reportada, los títulos o grados de sus académicos, etc.

Para complementar este análisis se realizaron también entrevistas semiestructuradas a funcionarios relevantes dentro de cada facultad. Con ello se buscó acceder a información menos directa, como pueden ser los criterios de asignación de la carga docente e investigativa (con su respectivo peso simbólico), la estructura de los sistemas de incentivos y la racionalidad detrás de ellos, las valoraciones de las actividades docentes e investigativas a la hora de organizar la promoción de la carrera académica, etc.

\subsubsection{Criterios de selección de la muestra por tipo de universidad}

Se buscó que los casos seleccionados abarcaran de la forma más amplia posible la diversidad de las instituciones chilenas en sus funciones docentes e investigativas. En este sentido, se ha argumentado que la diversidad del sistema educacional superior chileno no es bien aprehendida bajo la división de pertenencia o no pertenencia al Consejo de Rectores, pues esta segmentación no captura importantes diferencias tanto en la oferta programática como en otros factores relevantes (Mineduc, 2013). De hecho, no existe acuerdo en torno a qué clasificación universitaria puede resultar más útil a nivel internacional (Marginson, 2009). Sin embargo, una de las más aceptadas para el caso chileno es la de Rosso y Reyes (2012), basada 
en la Carnegie Classification, la cual divide el panorama respecto del volumen investigativo de sus instituciones y el número de programas de doctorado.

Ahora bien, al examinar de cerca el output investigativo del ámbito chileno se hace evidente que existen tres universidades cuya producción investigativa y cantidad de académicos y alumnos es ampliamente superior al resto de las instituciones del país, a saber, la Universidad de Chile, la Pontificia Universidad Católica de Chile y la Universidad de Concepción. Esta diferencia en volumen de publicación y tamaño absoluto da pie a la posibilidad de que en estas universidades la dinámica docencia-investigación se dé de manera distinta a las universidades que les siguen.

Por esta razón, se han considerado de gran utilidad los criterios de división esbozados por el Centro Interuniversitario de Desarrollo, los cuales se enfocan mayormente en la cantidad de publicaciones (Cinda, 2011). Por tanto, se ha buscado separar las universidades que, si bien podrían tener algún componente investigativo, son netamente docentes. Así, todas aquellas universidades que posean menos de 1.000 publicaciones indexadas según el Ranking Iberoamericano SIR 2011 realizado por Scimago Institutions Rankings en un periodo de cinco años, serán consideradas universidades docentes. Luego, las instituciones con más de 1.000 publicaciones, pero menos de 2.999 serán consideradas universidades con investigación, mientras que las universidades con más de 3.000 publicaciones serán consideradas universidades de investigación.

\subsubsection{Criterios de selección de la muestra por tipo de disciplina}

En cuanto a la división disciplinar, se ha definido la siguiente taxonomía de acuerdo con la manera en la que se vinculan la academia y la investigación (Gyarmati et al., 1984). En primer lugar, se tiene a las ciencias exactas (biología, química, matemática), las cuales están intimamente ligadas a la academia: su formación de pregrado está transversalmente determinada por un posterior trabajo en investigación. En segundo lugar, se tiene a las humanidades (historia, filosofía, letras), las cuales son similares a las ciencias exactas, en tanto que su formación también busca el desarrollo de habilidades de investigación. Sin 
embargo, sus metodologías son plenamente distintas, relacionadas con su divergencia temática y su carácter blando. En tercer lugar, se tiene a las carreras de profesión (medicina, derecho, pedagogía, arquitectura), las cuales tienen un conjunto de actividades socialmente esperadas y definidas relacionadas con su aplicabilidad. Por tanto, su vínculo con la investigación es difuso, cuando no inexistente. Por último, se tiene a las ciencias sociales (sociología, ciencias políticas, economía), las cuales no tienen una única actividad o labor predeterminada, y tampoco una relación necesaria con la investigación (que sin embargo es más relevante que en las carreras de profesión).

\subsubsection{Selección de la muestra}

En relación con lo anterior, se ha decidido seleccionar tres universidades por cada una de las tres categorías de cantidad de investigación ya explicitadas. Para cada caso seleccionado se realizará un análisis a nivel de facultades, que serán tipificadas de acuerdo con nuestras cuatro áreas de conocimiento (ver Tabla 1). Vale decir, se buscó tener basalmente una carrera representando cada disciplina por cada tipo de universidad; pero además, por razones de comparabilidad, se intentó conseguir como mínimo que cada disciplina por tipo de institución fuera ejemplificada por dos universidades.

La selección de las universidades para cada categoría se realizó de acuerdo con criterios respecto de la calidad en sus programas, tradición y fortaleza institucional, además de facilidad de acceso y contacto.

Tabla 1: Universidades seleccionadas

\begin{tabular}{|c|c|c|c|c|}
\hline & \multicolumn{3}{|c|}{ Tipo de universidad } \\
\hline & & $\begin{array}{l}\text { Universidades de } \\
\text { investigación }\end{array}$ & $\begin{array}{l}\text { Universidades con } \\
\text { investigación }\end{array}$ & $\begin{array}{l}\text { Universidades } \\
\text { docentes }\end{array}$ \\
\hline \multirow{4}{*}{ Disciplina } & Ciencias exactas & \multirow{4}{*}{$\begin{array}{c}\text { Pontificia } \\
\text { Universidad } \\
\text { Católica de Chile, } \\
\text { Universidad de } \\
\text { Chile, Universidad } \\
\text { de Concepción }\end{array}$} & \multirow{4}{*}{$\begin{array}{c}\text { Universidad de } \\
\text { Santiago de Chile, } \\
\text { Universidad } \\
\text { Diego Portales, } \\
\text { Universidad Austral } \\
\text { de Chile }\end{array}$} & \multirow{4}{*}{$\begin{array}{c}\text { Universidad Finis } \\
\text { Terrae, Universidad } \\
\text { Mayor, Universidad } \\
\text { Tecnológica } \\
\text { Metropolitana }\end{array}$} \\
\hline & Humanidades & & & \\
\hline & Profesionales & & & \\
\hline & Ciencias sociales & & & \\
\hline
\end{tabular}

Fuente: Elaboración propia. 


\subsubsection{Características del campo}

La recolección de datos se llevó a cabo en un periodo de tres semanas en las nueve instituciones seleccionadas. En las universidades de investigación (1A, 1B, 1C), se realizaron entrevistas a cinco facultades de ciencias, dos de humanidades, dos de profesión y dos de ciencias sociales; en las instituciones con investigación (2A, 2B, 2C), se realizaron entrevistas a tres facultades de ciencias, una de humanidades, tres de profesión y dos de ciencias sociales; y en las docentes (3A, 3B, 3C), se realizaron entrevistas a tres facultades de ciencias, dos de profesión y una de ciencias sociales.

Tabla 2: Entrevistas realizadas

\begin{tabular}{l|c|c|c|c|c|c|c|c|c}
\hline & $1 \mathrm{~A}$ & $1 \mathrm{~B}$ & $1 \mathrm{C}$ & $2 \mathrm{~A}$ & $2 \mathrm{~B}$ & $2 \mathrm{C}$ & $3 \mathrm{~A}$ & $3 \mathrm{~B}$ & $3 \mathrm{C}$ \\
\hline Humanidades & & $\checkmark$ & $\checkmark$ & $\checkmark$ & & & & & \\
\hline Profesión & $\checkmark$ & $\checkmark$ & & $\checkmark$ & $\checkmark$ & $\checkmark$ & $\checkmark$ & $\checkmark$ & \\
\hline Ciencias sociales & & $\checkmark$ & $\checkmark$ & $\checkmark$ & & $\checkmark$ & $\checkmark$ & & \\
\hline Ciencias naturales & $\checkmark \checkmark$ & $\checkmark \checkmark$ & $\checkmark$ & $\checkmark \checkmark$ & $\checkmark$ & & $\checkmark \checkmark$ & & $\checkmark$ \\
\hline
\end{tabular}

Fuente: Elaboración propia.

En definitiva, como indica la Tabla 2, se realizaron 25 entrevistas en total, cumpliendo los mínimos presupuestos a nivel general, pero sin conseguir el mínimo para humanidades, ni variabilidad en ciencias sociales. Estas excepciones tienen distintos fundamentos. En primer lugar, entre universidades con investigación y universidades docentes fue recurrente que las facultades de humanidades agruparan carreras que quedan fuera de nuestra taxonomía, o aún más, que concentraran misceláneamente todas aquellas carreras que no tienen una correspondencia clara con el resto de las áreas. A esto se agrega que en cada uno de estos niveles de menor o mínima investigación hay una universidad que no tiene facultad de humanidades, situación que solo apareció una vez avanzada la investigación, habiendo definido aquellas instituciones idóneas para realizarla. A lo anterior se agregan las evidentes complicaciones de acceso, dada la jerarquía y obligaciones de los cargos en cuestión. 


\section{Resultados}

\subsection{Descripción de los casos}

\subsubsection{Universidades de investigación}

Las universidades de investigación denotan un tamaño claramente superior al del resto de la muestra, reflejado en el tamaño de su matrícula anual, el número de programas y su amplio cuerpo académico. Además, cuentan con un alto porcentaje de contratos de jornada completa (ver Tabla 3).

Al mismo tiempo, al contar ya con al menos 70 años al momento de la reforma de 1981, les ha sido fácil adoptar el nuevo régimen y su exigencia de establecer la línea investigativa como piedra fundamental de actividad.

Tabla 3: Caracterización universidades de investigación

\begin{tabular}{|c|c|c|c|}
\hline & $1 \mathrm{~A}$ & $1 \mathrm{~B}$ & $1 \mathrm{C}$ \\
\hline Número de programas* & 67 pre 35 dip 278 pos & 91 pre 30 dip 112 pos & 61 pre 24 dip 175 pos \\
\hline Alumnos por universidad & 27.190 & 23.468 & 22.992 \\
\hline $\begin{array}{l}\text { Proporción de jornadas } \\
\text { completas por } \\
\text { universidad** }\end{array}$ & $\begin{array}{c}J C 1.782 \mathrm{JH} 1.185 \\
(60,1 \% \mathrm{JC})\end{array}$ & $\begin{array}{c}\text { JC } 1.336 \mathrm{JH} 212 \\
(86,3 \% \mathrm{JC})\end{array}$ & $\begin{array}{c}J C 1.959 \mathrm{JH} 1.172 \\
(62,6 \% \mathrm{JC})\end{array}$ \\
\hline Facultades entrevistadas & $\begin{array}{l}\text { Química, Cs. } \\
\text { Forestales y } \\
\text { Veterinaria }\end{array}$ & $\begin{array}{c}\text { Química, Cs. } \\
\text { Forestales, Economía, } \\
\text { Humanidades, } \\
\text { Arquitectura }\end{array}$ & $\begin{array}{c}\text { Química, Letras, } \\
\text { Economía }\end{array}$ \\
\hline Tamaño facultades & $1.500,315$ y 1.100 & $\begin{array}{c}500,560,1.000 \\
500 \text { y } 760\end{array}$ & 800,488 , y 2.300 \\
\hline $\begin{array}{l}\text { Proporción de académicos } \\
\text { en jornada completa por } \\
\text { facultades** }\end{array}$ & $90 / 10$ & $80 / 20$ & $80 / 20$ \\
\hline
\end{tabular}

Fuente: Elaboración propia.

${ }^{*}$ pre=pregrados, dip=diplomados, pos=posgrados

** Jornada completa = Media jornada o superior

\subsubsection{Universidades con investigación}

Como indica la Tabla 4, las universidades seleccionadas en este nivel tienen un tamaño menor que las de investigación, situación evidente en su matrícula y cantidad de programas de estudio (exceptuando 
la 2B). La diferencia es significativa en posgrado, ya que ninguna de estas instituciones ofrece más de 90 programas. En general, se trata de universidades creadas posteriormente a la reforma, por lo que su actividad en investigación es más baja en magnitud, aun cuando su crecimiento relativo es similar (Conicyt, 2014). Dada la menor dimensión de número de alumnos, número de programas y número de investigaciones, la cantidad de profesores jornada completa en términos absolutos también es menor a las primeras universidades, y en dos de ellas su proporción es menor a un tercio de los académicos totales.

Tabla 4: Caracterización universidades con investigación

\begin{tabular}{l|c|c|c}
\hline & $2 \mathrm{~A}$ & $2 \mathrm{~B}$ & $2 \mathrm{C}$ \\
\hline Número de programas & 54 pre 8 dip 50 pos & 96 pre 97 dip 81 pos & 34 pre 53 dip 36 pos \\
\hline Alumnos universidad & 11.646 & 19.763 & 13.167 \\
\hline $\begin{array}{l}\text { Proporción de jornadas } \\
\text { completas por universidad }\end{array}$ & $\begin{array}{c}\text { JC } 950 \mathrm{JH} 295(76,3 \% \\
\mathrm{JC})\end{array}$ & $\begin{array}{c}\text { JC } 663 \mathrm{JH} 1.295 \\
(33,9 \% \mathrm{JC})\end{array}$ & $\begin{array}{c}\text { JC } 469 \mathrm{JH} 1.269(27 \% \\
\mathrm{JC})\end{array}$ \\
\hline Facultades entrevistadas & $\begin{array}{c}\text { Cs. Forestales, } \\
\text { Ciencias, Economía, } \\
\text { Humanidades, } \\
\text { Medicina }\end{array}$ & Medicina, Ciencias & $\begin{array}{c}\text { Derecho y Ciencias } \\
\text { sociales }\end{array}$ \\
\hline Tamaño facultades & $\begin{array}{c}350,680,870,2.000 \\
\text { y 2.425 }\end{array}$ & 1.800 y 1.100 & 1.200 y 900 \\
\hline $\begin{array}{l}\text { Proporción de académicos } \\
\text { en jornada completa por } \\
\text { facultades }\end{array}$ & $90 / 10$ & $50 / 50$ & $40 / 60$ \\
\hline
\end{tabular}

Fuente: Elaboración propia.

\subsubsection{Universidades docentes}

Estas son de un tamaño evidentemente menor a las de los dos primeros niveles. Como señala la Tabla 5, si bien la mayor de ellas posee 15.000 matrículas anuales, las otras dos instituciones solo bordean las 4.000 y 7.000. Paralelamente, los programas ofrecidos en pregrado varían desde 27 a 87, y los de posgrado desde 5 a 87, siendo estos rangos mayores y con promedios más bajos que los de las universidades con investigación. En contraste, la cantidad absoluta de académicos con los que se cuenta no difiere en demasía respecto de las otras instituciones. Sin embargo, la proporción de ellos que forma parte de un cuerpo de planta o jornada completa en relación con profesores contratados por hora es radicalmente más baja. 
En cuanto a su tradición, las tres instituciones son relativamente recientes. Dos de ellas son producto de proyectos fundados a finales de la década de 1980, mientras que la tercera emerge como desprendimiento de una universidad estatal con amplia tradición institucional, situación ocurrida a principios de la década de 1990.

Tabla 5: Caracterización universidades docentes

\begin{tabular}{|c|c|c|c|}
\hline & $3 \mathrm{~A}$ & $3 B$ & $3 C$ \\
\hline Número de programas & 87 pre, 87 pos & 27 pre, 50 dip, 16 pos & 57 pre, 6 dip, 5 pos \\
\hline Alumnos universidad & 15.725 & 4.124 & 7.143 \\
\hline $\begin{array}{l}\text { Proporción de jornadas } \\
\text { completas por universidad }\end{array}$ & $\begin{array}{c}\text { JC } 624 \mathrm{JH} 2.241 \\
(21,8 \% \mathrm{JC})\end{array}$ & $\begin{array}{c}\text { JC } 168 \mathrm{JH} 841 \text { (16,7\% } \\
\mathrm{JC})\end{array}$ & $\begin{array}{c}\text { JC } 188 \mathrm{JH} 533(26,1 \% \\
\text { JC) }\end{array}$ \\
\hline Facultades entrevistadas & $\begin{array}{c}\text { Ciencias, Cs. } \\
\text { Forestales, } \\
\text { Economía, Medicina }\end{array}$ & Pedagogía & Química \\
\hline Tamaño facultades & $\begin{array}{c}100,2.000,1.200 y \\
5.800\end{array}$ & 400 & 400 \\
\hline $\begin{array}{l}\text { Proporción de académicos } \\
\text { en jornada completa por } \\
\text { facultades }\end{array}$ & $40 / 60,30 / 70$ & $10 / 90$ & $90 / 10$ \\
\hline
\end{tabular}

Fuente: Elaboración propia.

\subsection{Resultados por tipo de universidad}

\subsubsection{Universidades de investigación}

a. Definiciones de investigación y de docencia

En cuanto al posicionamiento formal de la investigación y docencia a nivel institucional, las tres universidades presentaron un discurso similar. Todas las documentaciones públicas declararon tanto a la enseñanza como a la investigación como las actividades primordiales de la entidad. Adicionalmente, las autoridades entrevistadas refirieron directamente a la tradición institucional, enfatizando que el rol de sus universidades siempre se ha enmarcado en un compromiso social de formación de personas, y que si bien la generación de conocimiento es valorable, la docencia es lo fundamental. 


\section{b. Distribución de actividades}

Como indica la Tabla 6, las cargas académicas jamás descienden bajo el nivel de dos o tres cursos semestrales, además de al menos un proyecto investigativo propio o un fondo nacional adjudicado. Con esto, cada una de estas actividades concentra el $40 \%$ de la carga horaria de sus académicos. Esto se debe principalmente a que existen ciertos lineamientos institucionales, que delimitan de forma clara las exigencias de los perfiles de la carrera académica.

Específicamente, las tres instituciones cuentan con una carrera académica principal bien definida, llamada categoría ordinaria, en donde se inserta la mayoría de los académicos de jornada completa. Ella generalmente exige que los profesores desarrollen un perfil integral, complementando labores de docencia, investigación, extensión y administración, aunque sus roles irrenunciables son los dos primeros.

Paralelamente, existe una carrera adjunta, en donde se inserta la mayoría de los académicos con contrato por hora, cuyo desempeño se evalúa en función de una sola de las actividades mencionadas. Por último, en dos de las instituciones existe una categoría docente, cuyas exigencias mínimas se concentran principalmente en la docencia, la cual se complementa con extensión y administración a medida que se asciende en la jerarquía. Esto permite absorber una tensión generacional, pues existen ciertas cohortes de académicos prestigiosos cuya formación data de periodos anteriores a la reforma de 1981 y, que por lo tanto, valora sobremanera la docencia en desmedro de la investigación. Por medio de esta carrera paralela, dichos profesores se pueden mantener dentro de la institución. 
Tabla 6: Resultados universidades de investigación

\begin{tabular}{l|c|c|c}
\hline $\begin{array}{l}\text { Carrera } \\
\text { académica }\end{array}$ & $\begin{array}{c}\text { Categoría Ordinaria/ } \\
\text { Categoría Docente/ } \\
\text { Categoría Adjunta }\end{array}$ & $\begin{array}{c}\text { Cuerpo Ordinario/Cuerpo } \\
\text { Especial }\end{array}$ & $\begin{array}{c}\text { Categoría Ordinaria/ } \\
\text { Categoría Docente/ } \\
\text { Categoría Adjunta }\end{array}$ \\
\hline $\begin{array}{l}\text { Distribución de } \\
\text { actividades }\end{array}$ & $\begin{array}{c}\text { Académico ordinario } \\
\text { realiza ambas }\end{array}$ & $\begin{array}{c}\text { Académico ordinario } \\
\text { realiza ambas }\end{array}$ & $\begin{array}{c}\text { Académico ordinario } \\
\text { realiza ambas }\end{array}$ \\
\hline $\begin{array}{l}\text { Carga } \\
\text { académica }\end{array}$ & $\begin{array}{c}40 \% \text { doc } 40 \% \text { inv 20\% } \\
\text { demás. Dos cursos al } \\
\text { semestre más al menos } \\
\text { una investigación, no } \\
\text { necesariamente publicada }\end{array}$ & $\begin{array}{c}\text { 40\% doc } 40 \% \text { inv 20\% } \\
\text { demás. Dos 0 tres cursos } \\
\text { al semestre más al menos } \\
\text { una investigación }\end{array}$ & $\begin{array}{c}40 \% \text { doc } 40 \% \text { inv 20\% } \\
\text { demás. Cuatro cursos al } \\
\text { año, más al menos una } \\
\text { investigación o un fondo } \\
\text { adjudicado }\end{array}$ \\
\hline $\begin{array}{l}\text { Criterios de } \\
\text { promoción }\end{array}$ & $\begin{array}{c}\text { Integral. Ascenso refiere a } \\
\text { prestigio }\end{array}$ & $\begin{array}{c}\text { Integral. Ascenso refiere a a } \\
\text { prestigio }\end{array}$ & $\begin{array}{c}\text { Integral. Ascenso refiere a } \\
\text { prestigio }\end{array}$ \\
\hline Evaluación & $\begin{array}{c}\text { Encuesta docente - Horas } \\
\text { realizadas / Fondecyt- ISI }\end{array}$ & $\begin{array}{c}\text { Encuesta docente y Ev. de } \\
\text { pares / ISI - Fondecyt - } \\
\text { SciELO - Libros - Obras }\end{array}$ & $\begin{array}{c}\text { Encuesta docente y Ev. de } \\
\text { pares / Fondecyt - SciELO } \\
\text { - ISI - Libros }\end{array}$ \\
\hline Incentivos & $\begin{array}{c}\text { Asignación por } \\
\text { investigación - Asignación } \\
\text { de exclusividad de docencia }\end{array}$ & $\begin{array}{c}\text { Asignación por desempeño } \\
\text { integral - Asignación de } \\
\text { docente }\end{array}$ & $\begin{array}{c}\text { Premio de excelencia en } \\
\text { investigación - Premio de } \\
\text { docencia }\end{array}$ \\
\hline
\end{tabular}

Fuente: Elaboración propia.

\section{c. Criterios de promoción}

Los criterios de promoción dependen fuertemente de la jerarquía de las distintas carreras académicas, siendo en este nivel la carrera ordinaria la más relevante.

En general, sus categorías no presentan mayores variaciones (de forma ascendente: instructor, asistente, asociado y titular), y sus perfiles específicos reflejan en distinta medida la lógica de vinculación entre investigación y docencia. A medida que se avanza, se espera que los académicos adquieran renombre nacional o internacional por su investigación autónoma, desarrollen excelencia docente capaz de atraer alumnos y formar académicos, y conjuguen estas habilidades con servicios, extensión y administración.

En este sentido, un fenómeno específico de este tipo de universidades radica en que cualquier desempeño sobresaliente debe ser logrado siempre sobre la base de la carga académica mínima estipulada. Es decir, no existen facilidades que permitan a académicos de producción de excelencia liberar su tiempo de otros quehaceres. 
Incluso más, si es que los profesores cumplen un periodo y no logran la promoción, son desvinculados de la carrera, la facultad o la universidad.

Debe notarse, en adición, que la promoción académica cuenta con ciertas divergencias disciplinarias. En las ciencias naturales, por ejemplo, la promoción y el prestigio son accesibles predominantemente a través de productos investigativos. Esto permite que a medida que se avanza en la carrera académica, los docentes puedan liberarse de tareas de gestión o administración para dedicarse a la investigación, pero nunca de la docencia. Un caso opuesto lo constituyen las facultades de disciplinas de profesión, en donde la relevancia o prestigio de un académico no surge necesariamente de sus productos investigativos, sino de "actividades relevantes" (como pueden ser obras importantes, en el caso de arquitectura).

\section{d. Criterios de evaluación}

La evaluación de desempeño de los académicos se realiza principalmente en dos instantes distintos: durante la evaluación de las postulaciones a promoción y en las evaluaciones bienales del cumplimiento de convenios de desempeño ${ }^{6}$.

En estas instancias, la evaluación de la labor docente se realiza de forma relativamente estandarizada. Para ello se toman en cuenta las encuestas de evaluación docente realizadas por alumnos, que en general son consideradas válidas y confiables por parte de los departamentos; y además, evaluaciones de facultad, realizadas por autoridades y por pares académicos, generalmente profesores titulares. Aquellas comisiones toman generalmente en cuenta portafolios y currículos, cantidad y tipos de cursos realizados, y la cantidad de tesis dirigidas en pregrado y posgrado.

Por su parte, la investigación tiende a ser evaluada con distintos métodos, dependiendo de la disciplina. Las ciencias naturales, por un lado, tendieron a focalizarse en la cantidad de artículos publicados en

6 Los convenios de desempeño son aquellas instancias donde el académico negocia su carga, comprometiéndose a cumplir con ciertas actividades durante un periodo determinado. 
revistas ISI o SciELO, considerando ponderadores de impacto y de citación, o bien cantidad de fondos nacionales y/o locales adjudicados, dando mayor relevancia a proyectos Fondecyt. Por otro lado, las ciencias sociales también valoraron la cantidad de publicaciones en revistas de corriente principal, con la salvedad de que aquellas revistas son evaluadas de acuerdo a rankings propios y específicos del área. Además, estas disciplinas, en conjunto con las humanidades, apreciaron la producción de libros o capítulos de libros, los cuales requieren de mayor tiempo de elaboración. Las segundas, por último, también consideraron investigación a la producción creativa, como es el caso de obras artísticas originales.

Ahora bien, se apuntó constantemente a la dificultad de medir de forma efectiva la buena labor docente, en tanto que es difícil distinguir de manera clara una buena docencia de una excelente. Para ello, se evalúa principalmente la cantidad y la antigüedad de la docencia realizada. En contraste, dado que la investigación es más fácil de medir, normalmente es tomada como criterio fundamental para el ascenso. Así, la docencia se entiende como un mínimo obligatorio, mientras que la investigación es el valor agregado que define la movilidad.

\section{e. Incentivos}

Por último, en todas las instituciones existen desde el nivel central al menos un incentivo fuerte a la investigación (distinto a los incentivos por publicación) y otro incentivo importante a la docencia, aunque el primero es más cuantioso. Sin embargo, se señala constantemente que el mayor incentivo para realizar ambas actividades es la carrera académica misma.

\subsubsection{Universidades con investigación}

a. Definición de investigación y docencia

Las tres universidades de este nivel tienen una vocación más profesionalizante y docente que las primeras. Una, por ser heredera de la Universidad Técnica del Estado, con lo que su deber público busca poner a disposición de su estudiantado y de la sociedad el avance del conocimiento. Otra, por ser una universidad pequeña y 
regional, que busca potenciar la industria, la ciencia y el conocimiento locales. La última, por ser una universidad privada que por ley no puede acceder al AFD, pero que busca tener una incidencia en el desarrollo de políticas públicas a nivel país. En definitiva, todas tienen un arraigo local o nacional, por un deber autoconferido o por falta de posibilidades/motivaciones para ampliar sus horizontes.

\section{b. Distribución de actividades}

En este nivel, como indica la Tabla 7 , existen solo dos plantas de académicos en las tres universidades observadas: una adjunta relacionada únicamente con la docencia y otra académica relacionada con investigación, extensión y administración. En general, estas plantas se vinculan con el tipo de jornada de los profesores: quienes realizan varias actividades normalmente tienen jornada completa o más de media jornada.

Los mínimos de producción científica y docencia, aunque no formales a nivel de universidad, en los hechos corresponden a dos cursos por semestre y una publicación indexada en general. Esta informalidad responde al hecho de que las tres instituciones funcionan con convenios de desempeño entre sus académicos de planta y sus facultades, permitiéndoles cierta flexibilidad respecto de la docencia impartida y la producción científica. Esta flexibilidad es levemente mayor a aquella que se observa en el primer nivel, en la medida en que responde a criterios de sobreproducción de una de las actividades "anexas": investigación, extensión o gestión. 
Tabla 7: Resultados universidades con investigación

\begin{tabular}{|c|c|c|c|}
\hline & $2 \mathrm{~A}$ & $2 \mathrm{~B}$ & $2 C$ \\
\hline $\begin{array}{l}\text { Carrera } \\
\text { académica }\end{array}$ & $\begin{array}{c}\text { Planta ordinaria / Planta } \\
\text { adjunta }\end{array}$ & $\begin{array}{c}\text { Plata Regular / Planta } \\
\text { por hora }\end{array}$ & $\begin{array}{l}\text { Carrera académica / } \\
\text { Carrera docente }\end{array}$ \\
\hline $\begin{array}{l}\text { Distribución } \\
\text { de actividades }\end{array}$ & $\begin{array}{l}\text { Ordinarios realizan inv. } \\
\qquad y \text { doc. }\end{array}$ & $\begin{array}{l}\text { Ordinarios realizan inv. } \\
\text { y doc. }\end{array}$ & $\begin{array}{l}\text { Ordinarios realizan inv. } \\
\text { y doc. }\end{array}$ \\
\hline $\begin{array}{l}\text { Carga } \\
\text { académica }\end{array}$ & $\begin{array}{l}\text { Dos o tres cursos } \\
\text { semestrales y una } \\
\text { publicación ISI. Cierta } \\
\text { flexibilidad permitida }\end{array}$ & $\begin{array}{l}\text { Dos o tres cursos } \\
\text { semestrales y una } \\
\text { publicación ISI. Cierta } \\
\text { flexibilidad permitida }\end{array}$ & $\begin{array}{c}\text { Dos cursos semestrales } \\
\text { y un proyecto de } \\
\text { investigación en proceso. } \\
\text { Cierta flexibilidad } \\
\text { permitida }\end{array}$ \\
\hline $\begin{array}{l}\text { Criterios de } \\
\text { promoción }\end{array}$ & $\begin{array}{l}\text { Integral inclinada a } \\
\text { investigación }\end{array}$ & Integral & $\begin{array}{c}\text { Cualitativo independiente } \\
\text { de investigación }\end{array}$ \\
\hline Evaluación & $\begin{array}{l}\text { Encuesta Docente / } \\
\text { Productos de proceso }\end{array}$ & $\begin{array}{l}\text { Encuesta Docente y Ev. de } \\
\text { pares / ISI - Fondecyt - } \\
\text { SciELO - Libros - Obras }\end{array}$ & $\begin{array}{l}\text { Encuesta Docente - Ev de } \\
\text { pares / "cualitativo" }\end{array}$ \\
\hline Incentivos & $\begin{array}{c}\text { Asignación por } \\
\text { investigación, viajes a } \\
\text { congresos }\end{array}$ & $\begin{array}{l}\text { Incentivo por publicación, } \\
\text { viajes a congresos }\end{array}$ & $\begin{array}{l}\text { Incentivo por proyecto } \\
\text { / publicación. Viajes y } \\
\text { materiales. }\end{array}$ \\
\hline
\end{tabular}

Fuente: Elaboración propia.

\section{c. Criterios de promoción}

En este nivel las comisiones de promoción, que definen qué académicos son ascendidos en la carrera académica, deben lidiar con facultades sobresalientes en investigación, frente a otras que no están al mismo nivel y que de igual modo requieren de expectativas de promoción. Por lo mismo, los criterios para dicha promoción son variables transversalmente al interior de la universidad: dependen del ajuste que realizan las comisiones de asignación de puntaje o de jerarquía en pos de otorgar puntuaciones "equitativas" por el trabajo y los productos realizados. Estas comisiones buscan además que sus académicos sean integrales, aunque en general, se reconoce que ellas no han desarrollado criterios totalmente confiables para realizar una compensación a aquellas actividades de medición más opaca. Entre ellas, destacan las investigaciones que no tienen como producto publicaciones indexadas. Solo una de las universidades no considera esta actividad como un criterio relevante para el ascenso, decidiéndose exclusivamente por evaluaciones cualitativas a nivel de alumnos y de facultad. 


\section{d. Criterios de evaluación}

Estas universidades se basan fundamentalmente en encuestas realizadas por los alumnos para la evaluación de la docencia de pregrado, aunque no se expresa absoluta conformidad con este mecanismo. Se señala que no se han logrado desarrollar indicadores más certeros para el mismo fin. Así, se da como resultado un desmedro de las actividades de docencia en general, en la medida en que no se toman en consideración para la promoción. La universidad 2C agrega evaluaciones idiosincráticas, como son la evaluación de la antigüedad de los cursos otorgados, un portafolio, la cantidad de horas enseñadas, un reporte completo de las actividades e intereses, entrevistas, proyecciones futuras, autoevaluación, etc.

\section{e. Incentivos}

Finalmente, estas universidades no tienen incentivos a la docencia a nivel institucional que sean relevantes (los incentivos existentes son escuetos y se relacionan con productos de esta actividad y asistencias a congresos) frente a los existentes para investigación. Estos corresponden a incentivos monetarios, que van desde los 500 mil a los 2 millones de pesos por publicación indexada; y de difusión, que sería el pago de viajes a congresos, la traducción e impresión de publicaciones, etc. Estos estímulos plantean una disyuntiva: dado el hecho de que estas universidades no son financiadas preponderantemente con recursos externos (AFD por publicaciones ISI y Fondecyt adjudicados), sino que con las mensualidades de sus alumnos y el AFI, al menos formalmente debieran estar en búsqueda de promocionar una docencia de la mejor calidad posible.

\subsubsection{Universidades docentes}

a. Definición de investigación y docencia

Las tres instituciones de este nivel han declarado formalmente dar espacio tanto a la investigación como a la docencia, pero en la práctica han focalizado su actividad en la segunda. En esta línea, las tres universidades entienden su rol principal en torno a la formación y profesionalización de sus estudiantes, en donde la posibilidad de investigación emerge siempre en función del desarrollo de habilidades 
valoradas en el mercado laboral. Solo una de estas instituciones ha mostrado indicios de avance hacia la producción de investigación científica publicable.

\section{b. Distribución de actividades}

Las actividades de los académicos suelen distribuirse tajantemente. En primer lugar, se atiende a la demanda de docencia generada por los alumnos de pregrado principalmente a través de la contratación de profesores por hora para la realización de un curso al semestre. Así, como se observa en la Tabla 8, existe una baja proporción de profesores con jornada completa, los cuales realizan más de una cátedra en la mayoría de los casos, y que además dedican parte importante de su tiempo a la administración. Solo en dos facultades entrevistadas se mencionó la existencia de una proporción de profesores con jornada completa que reparten equilibradamente sus labores de docencia e investigación. Por último, y contrario a lo anterior, no pocas facultades de la muestra indicaron la existencia de un grupo muy minoritario de investigadores full time que realiza docencia solo circunstancialmente, los cuales se encuentran ligados a centros de investigación independientes o incluso previos a la existencia de las facultades en cuestión. 
Tabla 8: Resultados universidades docentes

\begin{tabular}{|c|c|c|c|}
\hline & $3 \mathrm{~A}$ & $3 \mathrm{~B}$ & $3 C$ \\
\hline $\begin{array}{l}\text { Carrera } \\
\text { académica }\end{array}$ & $\begin{array}{l}\text { No existente, en creación } \\
\text { o dependiente de } \\
\text { credenciales externas }\end{array}$ & $\begin{array}{l}\text { Dependiente de } \\
\text { credenciales externas }\end{array}$ & $\begin{array}{l}\text { Planta ordinaria / Planta } \\
\text { adjunta }\end{array}$ \\
\hline $\begin{array}{l}\text { Distribución } \\
\text { de } \\
\text { actividades }\end{array}$ & $\begin{array}{l}\text { Académico JC: docencia } \\
\text { y administración, JP: } \\
\text { docencia + Cuerpo de } \\
\text { investigadores exclusivos }\end{array}$ & $\begin{array}{l}\text { Casi puramente docencia } \\
\text { y ocasionalmente } \\
\text { administración }\end{array}$ & $\begin{array}{l}\text { Docencia y otra actividad } \\
\text { a elección: investigación, } \\
\text { extensión o administración }\end{array}$ \\
\hline $\begin{array}{l}\text { Carga } \\
\text { académica }\end{array}$ & $\begin{array}{l}90 \% \text { a } 100 \% \text { de } \\
\text { docencia. Flexibilidad } \\
\text { para investigadores y } \\
\text { administradores }\end{array}$ & $\begin{array}{l}\text { Un curso al semestre. } \\
\text { Facultades con } \\
\text { investigación permiten } \\
\text { flexibilidad en docencia }\end{array}$ & $\begin{array}{l}\text { Mínimo 20\% de docencia } \\
\text { o un curso al semestre. } \\
\text { Quienes realizan proyectos } \\
\text { Fondecyt pueden recibir } \\
\text { rebaja en carga docente }\end{array}$ \\
\hline $\begin{array}{l}\text { Criterios de } \\
\text { promoción }\end{array}$ & $\begin{array}{l}\text { Docencia muy por sobre la } \\
\text { investigación }\end{array}$ & $\begin{array}{l}\text { Docencia, permanencia } \\
\text { en el grado y/o ejercicio } \\
\text { profesional destacado }\end{array}$ & $\begin{array}{l}\text { Actividad sostenida } \\
\text { en investigación y } \\
\text { competencias docentes: } \\
\text { comisión de promoción } \\
\text { (criterios laxos). } \\
\text { Permanencia en el cargo } \\
\text { como criterio fijo }\end{array}$ \\
\hline Evaluación & $\begin{array}{l}\text { Evaluación de estudiantes, } \\
\text { evaluación de facultad } \\
\text { bienal / Revista y número } \\
\text { de publicaciones }\end{array}$ & $\begin{array}{l}\text { Evaluación de estudiantes, } \\
\text { autoevaluaciones, política } \\
\text { de puertas abiertas }\end{array}$ & $\begin{array}{l}\text { Evaluación de estudiantes. } \\
\text { Comisión de profesores: } \\
\text { tesis dirigidas y } \\
\text { presentaciones en } \\
\text { congresos }\end{array}$ \\
\hline Incentivos & $\begin{array}{l}\text { Incentivo por proyectos } \\
\text { internos. Incentivo por } \\
\text { publicación ISI a nivel de } \\
\text { universidad: } 1 \text { a } 4 \text { millones } \\
\text { / Clases de posgrado, } \\
\text { asistencia a congresos }\end{array}$ & $\begin{array}{l}\text { Docencia: diplomados y } \\
\text { magísteres gratuitos en la } \\
\text { universidad, cursos en el } \\
\text { extranjero }\end{array}$ & No hay \\
\hline
\end{tabular}

Fuente: Elaboración propia.

\section{c. Criterios de promoción}

Los factores que determinan la promoción de la carrera académica varían en su claridad y consistencia a través de la muestra. En aquellas instituciones con mayor proporción de profesores de jornada por hora, los criterios de promoción son los mismos tanto para ellos como para profesores de planta, en la medida en que la clasificación está enfocada en la contratación y recontratación semestral y, por lo mismo, se basan en cuestiones externas, a saber: procedencia de los grados y posgrados, cantidad de años dedicados a la docencia 
universitaria, años de actividad profesional destacada, cantidad de cursos de especialización, grado académico en otras universidades, etc. Si bien existen criterios que se apoyan en la calidad docente o investigativa, como lo son las encuestas de evaluación docente por parte de los estudiantes o la publicación en revistas indexadas, ellos no conforman lo medular de la jerarquización.

Aquella institución derivada de la universidad estatal se desvía de esta generalización. Allí sí existe una diferenciación entre planta ordinaria y planta adjunta, en donde la primera debe presentar un perfil más integral para su promoción. No obstante, las exigencias son muy poco claras. Tanto los tipos de productos investigativos aceptados como su cantidad varían en un rango muy amplio. Incluso, algunos profesores cumplen su labor investigativa por medio de la guía de tesis o tesinas. El único criterio definido explícitamente son los años en el cargo.

\section{d. Criterios de evaluación}

Existen instancias de evaluación docente por parte de los alumnos, así como comisiones de facultad que miden la calidad docente en evaluaciones bienales. Pero, como ya se explicitó más arriba, el cuerpo principal de aquella instancia lo componen factores externos a la enseñanza. Por otro lado, la investigación cuenta con una amplia valoración a la hora de la evaluación, pero no existen pautas concretas para determinar su calidad o su impacto.

\section{e. Incentivos}

Por último, los incentivos a nivel institucional divergen dependiendo de la universidad. En uno de los establecimientos, en el cual hay mayores indicios de potenciar la investigación, existen incentivos claros a la publicación en revistas indexadas y a la concreción de proyectos internos y externos. Fuera de ello, sin embargo, en todo el nivel no existen estímulos adicionales importantes a ninguna de las dos actividades, más allá de la posibilidad de cursar diplomados o magísteres relacionados con el desarrollo de las capacidades docentes. 


\section{Análisis de resultados}

\subsection{Síntesis de resultados}

\subsubsection{Definiciones de investigación y docencia}

Respecto del planteamiento de las instituciones frente a las labores docentes e investigativas, existe una notoria diferencia en la conceptualización que se realiza en torno a la necesidad de llevar a cabo ambas actividades. Por un lado, las instituciones de investigación y con investigación plantean que las universidades en cuanto organizaciones tienen como mandato realizar ambas labores, mientras que las universidades del último nivel entienden que su papel principal descansa únicamente en la docencia. De esto se desprende que en el panorama chileno la docencia es un valor indiscutido.

Ahora bien, las diferencias se dan en cómo se entiende la investigación. En las universidades de investigación, la motivación está puesta en el deseo de ampliar las fronteras del conocimiento en un contexto global, mientras que en las universidades con investigación el impetu responde a la posibilidad de aplicación de dicho conocimiento en el contexto nacional o local. Esto se explica por el hecho de que las primeras corresponden a las universidades líderes del país según variados índices y, con ello, buscan insertarse en los círculos científicos mundiales a través de su amplio catálogo de publicaciones. Las segundas, por su parte, tienen un asentamiento local mucho más prominente, donde la generación de nuevos conocimientos busca la utilidad en el contexto social inmediato. Por último, las universidades docentes entienden la investigación como utilitaria para la generación de buenos profesionales, y no como un fin en sí mismo. Aquello que estas universidades recalcan como su labor es la formación de personas de acuerdo con un sello propio. Queda sin resolver el hecho de que, si es que estas universidades tuvieran los mismos recursos (sobre todo el acceso al AFD), prestigio o tamaño, volcarían su discurso hacia la investigación. 


\subsubsection{Distribución de actividades}

En general, la carga académica se relaciona con la forma en que la carrera académica define distintas distribuciones de actividad para diferentes plantas académicas. En este sentido, las universidades de investigación tienen una carrera ordinaria que determina una carga fuerte y equitativa de las distintas tareas, sin mayor posibilidad de flexibilizar estas responsabilidades. Este es el único nivel en el que ambas actividades se realizan a cabalidad. Por su parte, las universidades con investigación comparten la exigencia conjunta de actividades para la planta ordinaria, sin embargo, cuando hay desempeño sobresaliente en alguna de las áreas, se accede a una flexibilidad horaria que permite un mayor involucramiento en la actividad preferida. Por último, las universidades docentes no tienen una carrera académica definida, en tanto que sus profesores son contratados por horas para realizar cátedras. Dado que no existe más de una actividad, no hay una distribución de prácticas obligatorias que establecer. De hecho, los pocos académicos que realizan investigación lo hacen de forma exclusiva.

\subsubsection{Criterios de promoción}

En universidades de investigación, el insumo para el ascenso es la obtención de prestigio o renombre, el cual se consigue solamente mediante una destacada calidad en docencia e investigación. En este sentido, el desempeño de excelencia debe alcanzarse sobre la base de cumplir con las exigencias mínimas de carga académica y, por lo mismo, este éxito no conllevaría a una mayor flexibilidad. Por su parte, las universidades con investigación permiten una cierta flexibilidad, en la medida en que sus académicos sean sobresalientes en un área. Además, lidian con facultades destacadas en producción científica frente a otras de menor desempeño, lo que las obliga a equilibrar una estructura de promoción que abarque las posibilidades de todas las facultades. Esto lo consiguen a través de comités de asignación de puntaje, jerarquía y promoción. Por último, las universidades docentes, al no tener una carrera académica, definen sus grados académicos según juicios laxos, incluso llegando a considerar criterios externos. 


\subsubsection{Evaluación}

Las evaluaciones de docencia e investigación tienen distintos matices al comparar los tres niveles de la tipología. En universidades de investigación, por un lado, la docencia y la investigación se evalúan a través de encuestas a alumnos y dictámenes de comisiones de pares. Se constituyen además instancias para la evaluación del cumplimiento de convenios de desempeño y para evaluaciones de promoción. Estos mecanismos en general otorgan la posibilidad de aplicar criterios uniformes y relativamente exitosos en captar la calidad académica. Las universidades con investigación, en contraste, cuentan con evaluaciones docentes dadas por alumnos, aunque frecuentemente ellas son consideradas como indicadores poco certeros por las facultades. Al mismo tiempo, en algunas de estas universidades se cuenta con comisiones evaluadoras para el cumplimiento de convenios de desempeño, y otras comisiones para la postulación a promoción. En definitiva, en el nivel medio no existe un estándar uniforme de evaluación. Por último, en las universidades docentes la gran mayoría de las evaluaciones son realizadas por alumnos, o derechamente por instancias externas al desempeño.

\subsubsection{Incentivos}

Por último, los incentivos a las actividades varían en los distintos niveles. En el primero, dado el acceso a recursos y el planteamiento institucional, existen beneficios a ambas actividades, pero que dada la cantidad de académicos con jornada completa, son de menor magnitud que en universidades de otros niveles. En el nivel medio, en cambio, los esfuerzos se focalizan en mayor medida en el potenciamiento de la actividad investigativa, en desmedro de incentivos relacionados con el buen desempeño docente. Además, dado que existe una menor cantidad de académicos con jornada completa que podrían adjudicarse estos beneficios, ellos tienden a ser de mayor envergadura. Por último, el tercer nivel no presenta un patrón claro, en tanto que en una universidad hay incentivos fuertes a la investigación, en otra incentivos medios a la docencia, y en la última no existen incentivos en absoluto. 


\section{Conclusiones}

Tanto de la caracterización a nivel general, como respecto de los resultados levantados, es posible identificar en el caso chileno una estructuración del sistema universitario en línea con las tendencias mundiales de la NPM. Esta organización, concretamente bosquejada por el criterio de desempeño del AFD, pretende premiar a aquellas instituciones que tienen mejores capacidades, transfiriendo fondos según resultados fuertemente vinculados a la investigación. Este foco sobre dicha labor se debe a la facilidad con que sus productos y dividendos son cuantificados (Deem \& Lucas, 2007; Horta, Dautel \&Veloso, 2012; Jenkins, 2004; Marginson, 2006). Del mismo modo, este tipo de mecanismos se replica al interior de las universidades de cualquiera de los tres niveles, premiándose de manera más decisiva a las tareas de investigación, tanto por los incentivos que el sistema gubernamental otorga, como por las dificultades de evaluar la actividad docente.

Por otro lado, la acumulación de beneficios según el componente histórico del AFD pareciera cumplir una estratificación similar a la esbozada por Marginson (2006) respecto de las ventajas de las instituciones tradicionales y selectivas. De este modo, las universidades complejas serían las más capacitadas, al atraer los mayores recursos, los mejores académicos y los mejores alumnos, especialmente desde la reforma de los ochenta. Dada esta ventaja comparativa y estabilizada en el tiempo, las instituciones menos selectivas quedarían posicionadas en un nivel intermedio "de especialización" (que en el caso de Chile aparece como un enfoque de asentamiento local, como se indicó más arriba), o bien, relegadas a la exclusiva docencia (obligación que en Chile podría estar siendo ocultada por la retórica del sello valórico de los egresados).

En cuanto a la respuesta de las universidades ante el nuevo sistema central de financiamiento, podría decirse que su posicionamiento es diverso. En primer lugar, las universidades de investigación tienden a replicar de forma más cercana aquellos patrones de organización académica observados tradicionalmente a nivel internacional (Serow, 2000). Declarando discursiva y formalmente a la docencia y a la 
investigación como sus actividades fundamentales, han desarrollado una carrera académica exigente en ambos ámbitos. Sin embargo, al explorar de cerca sus métodos de evaluación, promoción e incentivos se hace evidente que son los productos investigativos tradicionales los que juegan el rol más relevante al momento de dirigir el accionar de los individuos. De este modo y, a pesar de que se esperaría que las recompensas del avance en la carrera académica reflejaran el discurso institucional (Diamond, 1993; Fairweather, 2005), la investigación ejerce un predominio innegable, lo que es consistente con otros casos internacionales (Serow, 2000; Deem \& Lucas, 2007; Jenkins, 2004).

Por su parte, en consistencia con lo planteado por Serow (2000), en estas universidades la docencia se conceptualiza como un mínimo al que se debe dar respuesta, y no como un ámbito en el que desarrollar excelencia. Así, estas instituciones cuentan con una gran proporción de académicos contratados a jornada completa, cumpliendo con estrictos estándares de actividad. Siguiendo a Marginson (2006), se puede especular que dicha exigencia puede ser fácilmente mantenida, en tanto que su posición privilegiada les permite contar con una alta oferta de académicos.

Por otra parte, las universidades con investigación demuestran funcionar también a través del imaginario ideal del académico investigador. Sin embargo, definen sus actividades en torno a realidades locales y específicas, por lo que existe mayor divergencia en la cristalización de sus lineamientos en exigencias concretas en la estructura de evaluación y recompensas. Aun así, cuentan con una carrera académica relativamente estructurada, aunque ella permite más flexibilidades y beneficios para aquellos que logran posicionarse como "publicadores" a nivel nacional, otorgándoles facilidades para disminuir su carga docente.

Por último, es en el nivel de las universidades docentes donde se presentan los rasgos que otorgan mayor especificidad a la realidad de educación terciaria chilena. En general, estas instituciones tienden a definir su misión en torno a la enseñanza, aunque queda inconcluso si ello sucede de manera forzada, al verse imposibilitadas de acceder a los fondos gubernamentales. En adición, presentan una organización 
de actividades sumamente anormal a las tendencias mundiales esbozadas por Teichler, Arimoto y Cummings (2013): cuentan con una proporción muy pequeña de académicos contratados a jornada completa, los cuales en general tienden a cumplir con tareas de docencia y servicio, e incluso a veces de investigación, pero cuya tarea primordial es la administración. En contraposición, existe en este grupo un número altísimo de académicos con contrato de media jornada, o incluso jornada por hora, que volátilmente se hacen cargo de la creciente demanda por docencia. Lo anterior impide la creación de una carrera académica estructurada, y sin ella, se desarrolla una alta variedad de instrumentos de medición de desempeño, que en general no se relacionan directamente con la calidad impartida.

En síntesis, Chile se ha presentado como un posible escenario en donde podrían desplegarse múltiples tensiones entre investigación y docencia en la organización de las actividades al interior de la universidad. Ello, debido a su inmersión en procesos de masificación, por un lado, y las reformas que privilegian la investigación, por el otro. Este artículo ha buscado ilustrar cómo se despliegan específicamente estas interacciones, enfocándose en las distintas dimensiones relacionadas con el quehacer académico. No obstante los resultados obtenidos, sería relevante indagar acerca de la experiencia de quienes se enfrentan a los distintos mecanismos, los propios académicos, en la medida en que los datos aquí recabados corresponden a la visión institucional, formal y normativa de la academia y sus organismos.

\section{Referencias}

Altbach, P. G., Reisberg, L. \& Rumbley, L. E. (2009). Trends in global higher education: Tracking an academic revolution. A report prepared for the Unesco 2009 World Conference on Higher Education. Recuperado de http://www.uis.unesco.org/Library/Documents/trends-global-highereducation-2009-world-conference-en.pdf

Auranen, O. \& Nieminen, M. (2010). University research funding and publication performance: An international comparison. Research Policy, 39(6), 822-834.

Bernasconi, A. y Rojas, F. (2003). Informe sobre la educación superior en Chile: 1980-2003. Santiago de Chile: Editorial Universitaria. 
Bernasconi, A. (2008). La profesionalización de la academia en Chile. Calidad en la Educación, 28, 15-27.

Bernasconi, A. (2010). La apoteosis del investigador y la institucionalización de la profesión académica en Chile. Estudios sobre Educación, 19, 139-163.

Berríos, P. (2008a). Carrera académica: análisis empírico de su estructura y organización en Chile. Calidad en la Educación, 29, 36-62.

Berríos, P. (2008b). Normas y percepciones sobre carrera académica en Chile. Calidad en la Educación, 28, 38-52.

Brunner, J. J. (1986). Informe sobre la educación superior en Chile. Santiago de Chile: FLACSO.

Centro Interuniversitario de Desarrollo, Cinda. (2010). El rol de las universidades en el desarrollo científico y tecnológico. Educación Superior en Iberoamérica, Informe 2010. Santiago de Chile: RIL editores.

Centro Interuniversitario de Desarrollo, Cinda. (2011). Educación Superior en Iberoamérica. Informe 2011. Santiago de Chile: RIL editores.

Centro Interuniversitario de Desarrollo, Cinda. (2012). Aseguramiento de la calidad en Iberoamérica. Informe 2012. Santiago de Chile: RIL editores.

Clark, B. R. (1997). The modern integration of research activities with teaching and learning. The Journal of Higher Education, 68(3), 241-255.

Clark, B. R. (1998). Creating entrepreneurial universities: Organizational pathways of transformation. Oxford: Pergamon Press.

Colbeck, C. L. (1998). Merging in a seamless blend: How faculty integrate teaching and research. Journal of Higher Education, 647-671

Comisión Nacional de Investigación Científica y Tecnológica, Conicyt. (2014). Artículos Wos (Ex-Isi) y SciELO de universidades chilenas 2000-2013. Recuperado de http://www.conicyt.cl/blog/2013/03/n\%C2\%BAarticulos-isi-universidades-cruch-y-privadas-2000-2011-3/

Contraloría General de la República. (2012). Financiamiento fiscal a la Educación Superior. Recuperado de http://www.contraloria.cl/ NewPortal2/portal2/ShowProperty/BEA\%20Repository/Merged/2013/ Archivos/146_Financiamiento_Fiscal_2012

De Boer, H., Enders, J. \& Schimank, U. (2007). On the way towards new public management? The governance of university systems in England, the Netherlands, Austria, and Germany. En D. Jansen (Ed.), New forms of governance in research Organizations (pp. 137-152). Dordretch: Springer International Publishing. 
Deem, R. \& Lucas, L. (2007). Research and teaching cultures in two contrasting UK policy contexts: Academic life in education departments in five English and Scottish universities. The International Journal of Higher Education and Educational Planning, 54(1), 115-133.

Diamond, R. M. (1993). Changing priorities and the faculty reward system. New Directions for Higher Education, 81, 5-12.

Enders, J., Kehm, B. M. \& Schimank, U. (2015). Turning universities into actors on quasi-markets: How new public management reforms affect academic research. En D. Jansen \& I. Pruisken (Eds.), The changing governance of higher education and research: Multilevel perspectives (pp. 89-103). Cham: Springer International Publishing.

Fairweather, J. S. (2005). Beyond the rhetoric: Trends in the relative value of teaching and research in faculty salaries. Journal of Higher Education, 76(4), 401-422.

Geiger, R. L. (2004). Knowledge and money: Research universities and the paradox of the marketplace. Stanford: Stanford University Press.

Griffiths, R. (2004). Knowledge production and the research-teaching nexus: The case of the built environment disciplines. Studies in Higher Education, 29(6), 709-726.

González, S., Brunner, J. J. y Salmi, J. (2013). Comparación internacional de remuneraciones académicas: un estudio exploratorio. Calidad en la Educación, 39, 22-42.

Gottlieb, E. E. \& Keith, B. (1997). The academic research-teaching nexus in eight advanced-industrialized countries. Higher Education, 34(3), 397-419.

Gyarmati, G., Gómez Millas, J., Browne, E., De la Barra, Á., Goic, A. y Guzmán, M. (1984). Las profesiones: dilemas del conocimiento y del poder. Santiago de Chile: Ediciones Universidad Católica de Chile.

Healey, M. (2005). Linking research and teaching exploring disciplinary spaces and the role of inquiry-based learning. Reshaping the university: new relationships between research, scholarship and teaching, 67-78.

Hirsch, F. (1976). Social limits to growth. Cambridge: Harvard University Press.

Horta, H., Dautel, V. \& Veloso, F. M. (2012). An output perspective on the teaching-research nexus: an analysis focusing on the United States higher education system. Studies in Higher Education, 37(2), 171-187.

Jenkins, A. (2004). A guide to the research evidence on teaching-research relations. Nueva York: The Higher Education Academy. 
Leisyte, L., Enders, J. \& De Boer, H. (2009). The balance between teaching and research in Dutch and English universities in the context of university governance reforms. Higher Education, 58, 619-635.

Link, A. N., Swann, C. A. \& Bozeman, B. (2008). A time allocation study of university faculty. Economics of Education Review, 27(4), 363-374.

Marginson, S. (2006). Dynamics of national and global competition in higher education. Higher education, 52(1), 1-39.

Marginson, S. (2009). The knowledge economy and higher education: Rankings and classifications, research metrics and learning outcomes measures as a system for regulating the value of knowledge. Higher Education Management and Policy, 21(1), 1-15.

Ministerio de Educación. (2013). Evidencias para políticas públicas en educación. Compilación Serie Evidencias 2012-2013. Santiago de Chile: Centro de Estudios Mineduc.

Organización para la Cooperación y el Desarrollo Económicos, OCDE. (2013). Revisión de politicas nacionales de educación: el aseguramiento de la calidad en la educación superior en Chile 2013. París: OECD Publishing.

Rosso, P. y Reyes, C. (Diciembre, 2012). Clasifying Chilean universities. En H., Beyer (Presidencia), Conferencia Internacional sobre Clasificación de Instituciones de Educación Superior. Conferencia llevada a cabo en División de Educación Superior, Ministerio de Educación, Santiago de Chile.

Teichler, U., Arimoto, A. \& Cummings, W. (2013). The changing academic profession: Major findings of a comparative survey. Dordrecht: Springer.

Serow, R. C. (2000). Research and teaching at a research university. Higher Education, 40(4), 449-463.

Recibido: 31/03/2015

Aceptado: 27/05/2015 